\title{
Renal cell carcinoma with tumor thrombus growing against the direction of venous return: an indicator of complicated surgery and poor prognosis
}

\section{Zhuo Liu}

Peking University Third Hospital

\section{Yuxuan Li}

Peking University Third Hospital

\section{Xun Zhao}

Peking University Third Hospital

\section{Liyuan Ge}

Peking University Third Hospital

\section{Guodong Zhu}

Peking University Third Hospital

\section{Peng Hong}

Peking University Third Hospital

\section{Shiying Tang}

Peking University Third Hospital

\section{Shudong Zhang}

Peking University Third Hospital

\section{Xiaojun Tian}

Peking University Third Hospital

\section{Shumin Wang}

Peking University Third Hospital

Cheng Liu

Peking University Third Hospital

Hongxian Zhang

Peking University Third Hospital

Lulin Ma ( $\square$ malulinpku@163.com )

Peking University Third Hospital

\section{Research Article}

Keywords: renal cell carcinoma, tumor thrombus, surgery, prognosis, growing against the direction of venous return 
Posted Date: April 16th, 2021

DOl: https://doi.org/10.21203/rs.3.rs-415158/v1

License: (c) (1) This work is licensed under a Creative Commons Attribution 4.0 International License. Read Full License

Version of Record: A version of this preprint was published at BMC Surgery on December 1st, 2021. See the published version at https://doi.org/10.1186/s12893-021-01448-0. 


\section{Abstract}

Purpose: To explore the effect of tumor thrombus growing against the direction of venous return (GADVR) tumor thrombus on the choice of surgical approach, the impact on the complexity of the surgery and the prognosis.

Methods: We retrospectively analyzed the clinicopathological data of 213 patients, who underwent surgery in a single center of Peking University Third Hospital between January 2016 and June 2020. For right renal cell carcinoma (RCC) and venous tumor thrombus (VTT), imaging revealed a filling defect in the left renal vein, which was significantly enhanced in enhanced imaging, suggesting that the tumor thrombus grew against the direction of venous return into the left renal vein. For left RCC and VTT, at least one of the left renal vein branches has tumor thrombus. The branches include the left adrenal vein, the left gonadal vein (testicular vein or ovarian vein), and the left ascending lumbar vein. The patients were divided into two groups according to whether they were GADVR tumor thrombus, and we compare the clinicopathological characteristics of GADVR tumor thrombus and non-GADVR tumor thrombus. Univariate and multivariate Cox regression analyses were performed to explore the risk factors that affect the prognosis of patients with RCC and VTT. Kaplan-Meier plots were conducted to evaluate the effect of GADVR on progression-free survival (PFS).

Results: Compared with non-GADVR tumor thrombus, patients with GADVR tumor thrombus had a higher proportion of open surgery (76.2\% vs. $52.1 \%, \mathrm{P}=0.035)$, a higher proportion of tumor thrombus adhering to the inferior vena cava (IVC) vessel wall ( $81 \%$ vs. $45.8 \%, \mathrm{P}=0.002)$, a higher proportion of segmental resection of the IVC vessel wall (61.9\% vs. $15.6 \%, \mathrm{P} \otimes 0.001)$; higher preoperative serum creatinine value $(110.0 \mu \mathrm{mol} / \mathrm{L}$ vs. $91.0 \mu \mathrm{mol} / \mathrm{L}, \mathrm{P}=0.015)$, a higher proportion of tumor thrombus combined with bland thrombus (non-tumor thrombus) $(57.1 \%$ vs. $19.8 \%, P<0.001)$. In terms of surgical complexity, patients with GADVR tumor thrombus had a longer median operation time ( $379.0 \mathrm{~min}$ vs. $308 \mathrm{~min}, \mathrm{P}=0.038$ ), more median surgical blood loss $(1400 \mathrm{ml}$ vs. $600 \mathrm{ml}, \mathrm{P}=0.018)$, and more postoperative complications $(52.4 \%$ vs. $30.7 \%, P=0.045)$. Multivariate Cox regression analysis showed that GADVR tumor thrombus, symptoms, postoperative serum creatinine, distant metastasis, sarcomatoid feature, pathological type, lymph node dissection were independent risk factors for PFS. Patients with GADVR tumor thrombus's median survival time was 14.0 months, while patients with non-GADVR tumor thrombus were 32.0 months $(P=0.016)$. GADVR tumor thrombus is an independent risk factor for PFS in patients with RCC and VTT.

Conclusion: GADVR tumor thrombus is a characteristic feature of tumor thrombus, with an incidence of $9.9 \%$. It has a higher proportion of open surgery and higher surgical complexity, which is an independent risk factor for PFS.

\section{Introduction}


Renal cell carcinoma (RCC) is a common malignant tumor of the urinary system. The incidence of RCC in China in 2015 was 3.35 per 100,000,(1) and in the past two decades, the incidence rate has increased by about $2 \%$ each year(2). One of the essential clinical features of RCC is that it often invades the renal vein and inferior vena cava (IVC) and forms venous tumor thrombus, which accounts for about $4-10 \%$ of RCC patients(3). The overall prognosis of patients with RCC and VTT is poor, and the 5-year tumor-specific survival rate is only $25-53 \%(4,5)$. Radical nephrectomy with thrombectomy is the only possible radical treatment for non-metastatic RCC with IVC tumor thrombus, but the 5-year survival rate is still $40-65 \%(6$, 7). Clarifying the growth characteristics of tumor thrombus has essential clinical value for diagnosing and treating this type of disease. According to the traditional view, the growth process of tumor thrombus in the IVC is as follows: primary renal tumor cells proliferate continuously, break through the capillary endothelial and enter the blood vessels, through the direction of venous return, grow along the affected renal vein and enter the IVC(8-10). Tumor thrombus in a few patients may enter the right atrium through the IVC $(11,12)$. However, in the clinical practice, we found a particular type of patients whose tumor thrombus grew against the direction of venous return. We defined it as Tumor thrombus growing against the direction of venous return (GADVR) tumor thrombus. The right renal vein usually has no extrarenal branches. The left renal vein receives the left adrenal vein, left testis, or ovarian vein. In some patients, the left renal vein is connected to the left ascending lumbar vein(13). For the right RCC and VTT, the GADVR tumor thrombus is manifested as the left renal vein tumor thrombus. For left RCC and VTT, the GADVR tumor thrombus showed left adrenal vein, left gonadal vein (testicular or ovarian vein), and left lumbar ascending vein tumor thrombus. We found that this GADVR tumor thrombus is rare in clinical practice, but its operation is more complex, and the prognosis is worse. For further validation, we retrospectively analyzed the clinicopathological data of 213 patients admitted to the single center from January 2016 to June 2020. They were divided into two groups according to whether they were GADVR tumor thrombus.

\section{Materials And Methods}

Inclusion criteria were as follows: (a) preoperative imaging examination, urinary enhanced CT, and/or IVC enhanced MRI showed RCC with renal vein or IVC tumor thrombus. (b)For right RCC and VTT, imaging revealed a filling defect in the left renal vein or right gonadal vein, which was significantly enhanced in enhanced imaging, suggesting that the tumor thrombus grew against the venous return into the left renal vein or the right gonadal vein. For left RCC and VTT, at least one of the left renal vein branches has tumor thrombus. The branches include the left adrenal vein, the left gonadal vein (testicular vein or ovarian vein), and the left ascending lumbar vein. In addition to imaging findings indicating GADVR tumor thrombus' presence, it is necessary to explore its presence during surgery and confirm the tumor thrombus by postoperative pathological diagnosis. (See Fig. 1). The exclusion criteria were as follows: (a) GADVR type tumor thrombus was considered preoperatively and confirmed by postoperative pathological diagnosis as bland thrombus (non-neoplastic). (b) Patients without surgery. (c)Postoperative pathological type was non-renal cell carcinoma. Patients' clinical manifestations were divided into local symptoms such as low back pain, hematuria, abdominal mass, and systemic symptoms such as fever and weight loss. The surgical methods were divided into laparoscopic minimally invasive approach and 
open approach. Tumor thrombus classification is divided into five categories according to the position of the proximal end of the tumor thrombus using Mayo classification.(14) Inferior vena cava MRI was used to determine whether the tumor thrombus was associated with bland thrombus (non-tumor thrombus), and the diagnostic criteria were based on our previous studies(15). All patients were routinely performed preoperative abdominal B-ultrasound and abdominal enhanced CT to determine whether there were liver metastasis and adrenal metastasis, and lung CT plain scan was performed to determine whether there was lung metastasis. For those with bone pain or central nervous system symptoms, bone scan or head MRI should be improved. For patients with acceptable economic conditions, PETCT can be improved to comprehensively evaluate systemic metastasis. The American Society of Anesthesiologists (ASA) score was used to evaluate the general condition before anesthesia.

Surgical method: for the IVC tumor thrombus: (a) simple tumor thrombus removal: after vascular occlusion, the IVC wall was cut to remove the tumor thrombus, then the IVC wall was sutured. (b) Inferior vena cava transection: the tumor thrombus did not invade the vascular wall, but there was an extended non-neoplastic bland thrombus at the tumor thrombus's distal end. After removing the tumor thrombus, the inferior vena cava was transected to prevent the bland thrombus from falling off.(16) (c) Segmental resection of the inferior vena cava: tumor thrombus invaded the vessel wall extensively, and the inferior vena cava was segmentally resected. Imaging diagnostic methods of tumor thrombus invasion of the vascular wall have been introduced in previous studies(17). For right RCC with GADVR tumor thrombus: (a) simple left renal vein tumor thrombus removal: tumor thrombus did not invade the left renal vein; 2 Segmental resection of the left renal vein: tumor thrombus invaded left renal vein and segmental resection of invaded left renal vein. For left RCC with GADVR tumor thrombus, the left renal vein branches involved by the tumor thrombus were resected segmentally. Local lymph node dissection was performed in patients with enlarged lymph nodes by preoperative imaging. Postoperative complications were performed using the Clavien-Dindo surgical complication classification method, among which grades $\geq$ three were defined as severe complications.(18)

Postoperative follow-up included survival, tumor recurrence or metastasis, postoperative renal function, and adjuvant drugs. Patients were asked to conduct an outpatient review every 3-6 months within two years after the operation, every 6-12 months after two years, and once a year after five years.

\section{Statistical analysis}

The normality of continuous variables was tested, and the data of normal distribution were expressed as mean \pm standard deviation; two independent samples T-test was used to analyze. For non-normal distribution data, they were expressed as median (IQR), using Mann-Whitney $U$ test analysis. Categorical data were expressed by frequency (percentage), using chi-square test, continuity correction, or Fisher's exact test was used if the chi-square test was not met. Univariate and multivariate Cox regression was performed to evaluate the prognostic significance of each variable relative to PFS. Kaplan-Meier plot was performed to evaluate the effect of GVDAR on PFS, and $p<0.05$ indicated that the difference was statistically significant. All statistical analyses were performed using SPSS 18.0. 


\section{Results}


Table 1

Comparison of clinicopathological features between GADVR tumor thrombus and non-GADVR tumor thrombus

\begin{tabular}{|c|c|c|c|}
\hline & non-GADVR(n = 192) & $\operatorname{GADVR}(n=21)$ & p \\
\hline Gender, n (\%) & $146(76.0 \%)$ & $16(76.3 \%)$ & 0.988 \\
\hline male & $46(24.0 \%)$ & $5(23.8 \%)$ & \\
\hline \multicolumn{4}{|l|}{ female } \\
\hline Age, y, median (IQR) & $60.00(14.00)$ & $59.00(10.00)$ & 0.550 \\
\hline Clinical symptoms, n (\%) & $50(26.0 \%)$ & $1(4.8 \%)$ & \multirow[t]{4}{*}{0.068} \\
\hline No & $94(49.0 \%)$ & $16(76.2 \%)$ & \\
\hline Local symptoms & $12(6.3 \%)$ & $1(4.8 \%)$ & \\
\hline Systemic symptoms & $36(18.8 \%)$ & $3(14.3 \%)$ & \\
\hline \multicolumn{4}{|l|}{ Both } \\
\hline $\mathrm{BMI}, \mathrm{kg} / \mathrm{m}^{2}$, mean $\pm \mathrm{SD}$ & $23.87 \pm 3.91$ & $24.95 \pm 2.66$ & 0.220 \\
\hline Surgical approach, n (\%) & $92(47.9 \%)$ & $5(23.8 \%)$ & \multirow[t]{3}{*}{0.035} \\
\hline Laparoscope & $100(52.1 \%)$ & $16(76.2 \%)$ & \\
\hline \multicolumn{3}{|l|}{ Open } & \\
\hline Mayo classification, n (\%) & $54(28.1 \%)$ & $2(9.5 \%)$ & \multirow[t]{6}{*}{0.093} \\
\hline 0 & $34(17.7 \%)$ & $3(14.3 \%)$ & \\
\hline 1 & $70(36.5 \%)$ & $12(57.1 \%)$ & \\
\hline 2 & $19(9.9 \%)$ & $4(19.0 \%)$ & \\
\hline 3 & $15(7.8 \%)$ & $0(0 \%)$ & \\
\hline \multicolumn{3}{|l|}{4} & \\
\hline IVC segmental resection, $\mathrm{n}(\%)$ & $162(84.4)$ & $8(38.1 \%)$ & \multirow{3}{*}{$\dot{0} 001$} \\
\hline No & $30(15.6)$ & $13(61.9 \%)$ & \\
\hline Yes & & & \\
\hline $\begin{array}{l}\text { Preoperative serum creatinine, } \mu \mathrm{mol} / \mathrm{L} \text {, } \\
\text { median (IQR) }\end{array}$ & $91.00(27.00)$ & $110.00(34.00)$ & 0.015 \\
\hline
\end{tabular}

GADVR: growing against the direction of venous return; IQR: interquartile range; BMI: body mass index; SD: standard deviation; RCC: renal cell carcinoma; ASA: American Society of Anesthesiologists; IVC: inferior vena cava; 


\begin{tabular}{|c|c|c|c|}
\hline & non-GADVR(n = 192) & $\operatorname{GADVR}(n=21)$ & p \\
\hline $\begin{array}{l}\text { Serum creatinine one week after operation, } \\
\mu \mathrm{mol} / \mathrm{L} \text {, median (IQR) }\end{array}$ & $97.00(33.00)$ & $96.00(47.00)$ & 0.739 \\
\hline Side, n (\%) & $73(38.0 \%)$ & $10(47.6 \%)$ & 0.392 \\
\hline Left & $119(62.0 \%)$ & $11(52.4 \%)$ & \\
\hline \multicolumn{4}{|l|}{ Right } \\
\hline Tumor diameter, $\mathrm{cm}$, mean $\pm \mathrm{SD}$ & $8.94 \pm 3.15$ & $8.42 \pm 2.52$ & 0.472 \\
\hline Clinical N stage, n (\%) & $79(41.1 \%)$ & $5(23.8 \%)$ & 0.123 \\
\hline cNO & $113(58.9 \%)$ & $16(76.2 \%)$ & \\
\hline \multicolumn{4}{|l|}{$\mathrm{cN} 1$} \\
\hline Adrenal metastasis, $\mathrm{n}(\%)$ & $176(91.7 \%)$ & $18(85.7 \%)$ & 0.613 \\
\hline No & $16(8.3 \%)$ & $3(14.3 \%)$ & \\
\hline \multicolumn{4}{|l|}{ Yes } \\
\hline Distant metastasis, n (\%) & $136(70.8 \%)$ & $17(81.0 \%)$ & 0.328 \\
\hline No & $56(29.2 \%)$ & $4(19.0 \%)$ & \\
\hline \multicolumn{4}{|l|}{ Yes } \\
\hline Bland thrombus, n (\%) & $154(80.2 \%)$ & $9(42.9 \%)$ & \multirow{3}{*}{$\begin{array}{l}<.001 \\
0.001\end{array}$} \\
\hline No & $38(19.8 \%)$ & $12(57.1 \%)$ & \\
\hline \multicolumn{3}{|l|}{ Yes } & \\
\hline Sarcomatoid feature, n (\%) & $167(87.0 \%)$ & $18(85.7 \%)$ & 1.000 \\
\hline No & $25(13.0 \%)$ & $3(14.3 \%)$ & \\
\hline \multicolumn{4}{|l|}{ Yes } \\
\hline Perirenal fat infiltration, $\mathrm{n}(\%)$ & $130(67.7 \%)$ & $15(71.4 \%)$ & 0.728 \\
\hline No & $62(32.3 \%)$ & $6(28.6 \%)$ & \\
\hline \multicolumn{4}{|l|}{ Yes } \\
\hline Pathology type, n (\%) & $160(83.3 \%)$ & $17(81.0 \%)$ & 1.000 \\
\hline Clear cell RCC & $32(16.7 \%)$ & $4(19.0 \%)$ & \\
\hline \multicolumn{4}{|l|}{ Non-clear cell RCC } \\
\hline $\begin{array}{l}\text { GADVR: growing against the direction of ver } \\
\text { index; SD: standard deviation; RCC: renal cel } \\
\text { IVC: inferior vena cava; }\end{array}$ & $\begin{array}{l}\text { eturn; IQR: interquart } \\
\text { noma; ASA: America }\end{array}$ & $\begin{array}{l}\text { ange; BMI: body I } \\
\text { ociety of Anesth }\end{array}$ & $\begin{array}{l}\text { s } \\
\text { ogists; }\end{array}$ \\
\hline
\end{tabular}




\begin{tabular}{|c|c|c|c|}
\hline & non-GADVR(n = 192) & $\operatorname{GADVR}(\mathrm{n}=21)$ & $\mathbf{p}$ \\
\hline Nuclear grade, $\mathrm{n}(\%)$ & $3(1.6 \%)$ & $0(0 \%)$ & 0.528 \\
\hline 1 & $70(36.5 \%)$ & $8(38.1 \%)$ & \\
\hline 2 & $76(39.6 \%)$ & $11(52.4 \%)$ & \\
\hline 3 & $43(22.4 \%)$ & $2(9.5 \%)$ & \\
\hline \multicolumn{4}{|l|}{4} \\
\hline Lymph node dissection, $\mathrm{n}(\%)$ & $105(54.7 \%)$ & $12(57.1 \%)$ & 0.830 \\
\hline No & $87(45.3 \%)$ & $9(42.9 \%)$ & \\
\hline \multicolumn{4}{|l|}{ Yes } \\
\hline ASA grade, $\mathrm{n}(\%)$ & $13(6.8 \%)$ & $2(9.5 \%)$ & 0.816 \\
\hline 1 & $156(81.3 \%)$ & $17(81.0 \%)$ & \\
\hline 2 & $23(12.0 \%)$ & $2(9.5 \%)$ & \\
\hline \multicolumn{4}{|l|}{3} \\
\hline Operative time, min, median (IQR) & $308.00(158.00)$ & $379.00(138.00)$ & 0.038 \\
\hline Surgical blood loss, mL, median (IQR) & $600.00(1550.00)$ & $1400.00(850.00)$ & 0.018 \\
\hline Postoperative complication, n (\%) & $133(69.3 \%)$ & $10(47.6 \%)$ & 0.045 \\
\hline No & $59(30.7 \%)$ & $11(52.4 \%)$ & \\
\hline \multicolumn{4}{|l|}{ Yes } \\
\hline Severe postoperative complication, n (\%) & $180(93.8 \%)$ & $19(90.5 \%)$ & 0.912 \\
\hline No & $12(6.3 \%)$ & $2(9.5 \%)$ & \\
\hline \multicolumn{4}{|l|}{ Yes } \\
\hline Thrombus adhering to the IVC, $\mathrm{n}(\%)$ & $104(54.2 \%)$ & $4(19.0 \%)$ & 0.002 \\
\hline No & $88(45.8 \%)$ & $17(81.0 \%)$ & \\
\hline \multicolumn{4}{|l|}{ Yes } \\
\hline $\begin{array}{l}\text { GADVR: growing against the direction of } \\
\text { index; SD: standard deviation; RCC: renal } \\
\text { IVC: inferior vena cava; }\end{array}$ & $\begin{array}{l}\text { eturn; IQR: interquart } \\
\text { inoma; ASA: America }\end{array}$ & $\begin{array}{l}\text { ange; BMI: body m } \\
\text { ociety of Anesthes }\end{array}$ & logists; \\
\hline
\end{tabular}

We retrospectively analyzed the clinical characteristics of 21 patients with GADVR tumor thrombus and 192 patients with non-GADVR tumor thrombus in our center. Compared with non-GADVR tumor thrombus, patients with GADVR tumor thrombus had a higher proportion of open surgery $(76.2 \%$ vs. $52.1 \%$, $\mathrm{P}=0.035)$, a higher proportion of tumor thrombus adhering to the inferior vena cava (IVC) vessel wall ( $81 \%$ 
vs. $45.8 \%, P=0.002)$, a higher proportion of segmental resection of the IVC vessel wall (61.9\% vs. $15.6 \%, P$ $\varangle 0.001)$; higher preoperative serum creatinine value $(110.0 \mu \mathrm{mol} / \mathrm{L}$ vs. $91.0 \mu \mathrm{mol} / \mathrm{L}, \mathrm{P}=0.015)$, a higher proportion of tumor thrombus combined with bland thrombus (non-tumor thrombus) $(57.1 \%$ vs. $19.8 \%$, $\mathrm{P}<0.001)$. In terms of surgical complexity, patients with GADVR tumor thrombus had a longer median operation time (379.0 min vs. $308 \mathrm{~min}, \mathrm{P}=0.038$ ) and more median surgical blood loss ( $1400 \mathrm{ml}$ vs. $600 \mathrm{ml}, \mathrm{P}=0.018$ ), and more postoperative complications ( $52.4 \%$ vs. $30.7 \%, \mathrm{P}=0.045)$. In other aspects, such as gender, age, clinical symptoms, BMI, Mayo classification, ASA grade, postoperative serum creatinine, tumor side, tumor diameter, lymph node metastasis and distant metastasis, pathological findings of sarcomatoid feature, perirenal fat infiltration, pathological type, nuclear grade, and severe postoperative complication, there was no significant difference between the two groups. (See Table 1) 
Table 2

Univariate and multivariate Cox regression analysis of prognostic factors for renal cell carcinoma with venous tumor thrombus

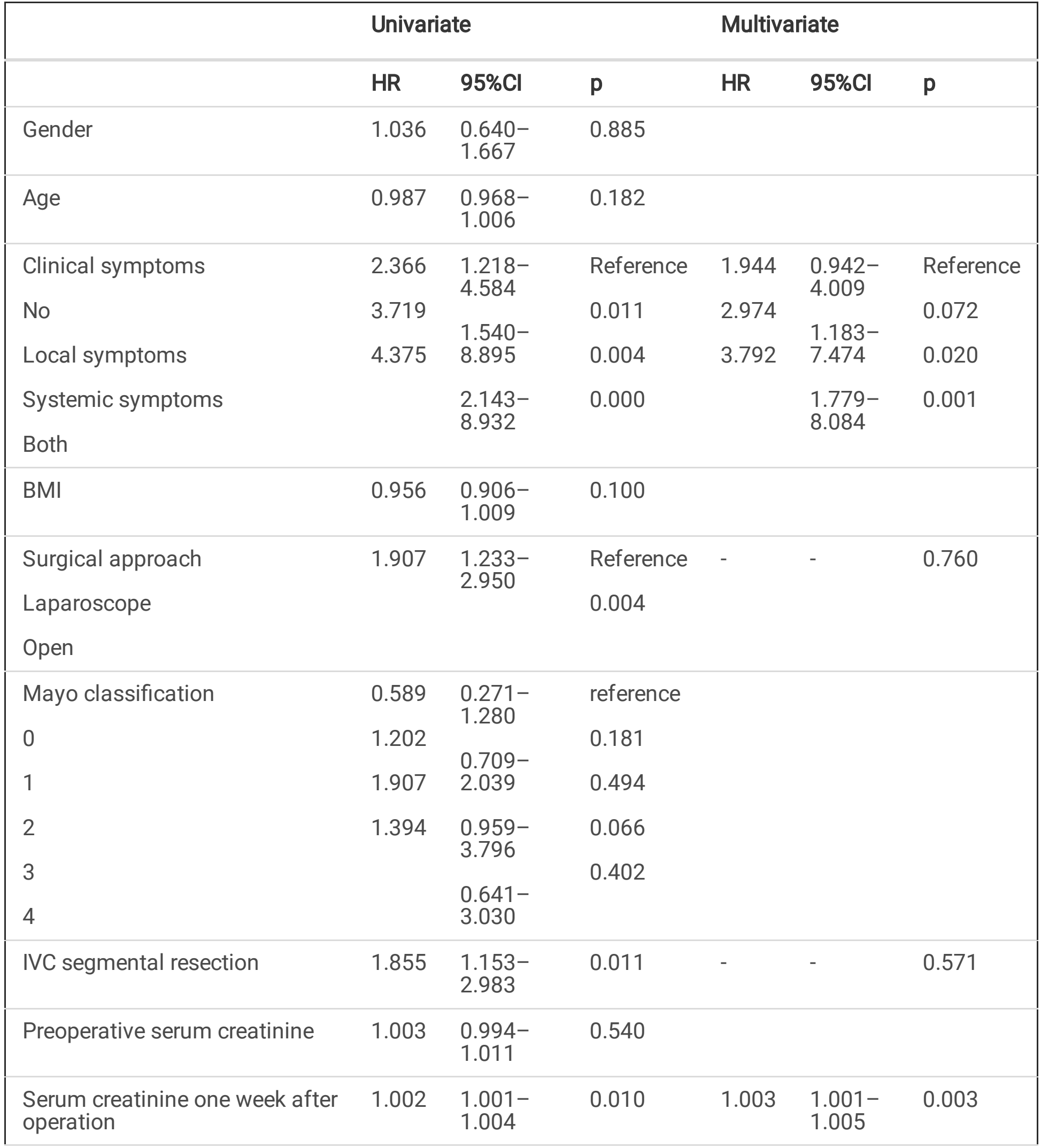

HR: Hazard ratios; Cl: confidence interval; BMI: body mass index; IVC: inferior vena cava; RCC: renal cell carcinoma; ASA: American Society of Anesthesiologists; GADVR: growing against the direction of venous return 


$\begin{array}{llll}\text { Side, } & 0.706 & 0.465- & \text { Reference } \\ \text { Left } & & 1.070 & 0.101\end{array}$

Right

$\begin{array}{llll}\text { Tumor diameter } & 1.059 & & 0.077 \\ \text { Clinical N stage } & 1.521 & 0.980- & \text { Reference } \\ \text { CNO } & & 2.359 & 0.061\end{array}$

cN1

Adrenal metastasis

1.425

0.737-

0.293

2.756

Distant metastasis

2.571

$1.690-$

$<0.001$

3.912

1.751

$1.120-$

0.014

2.571

$1.580-<0.001$

Bland thrombus

2.738

Sarcomatoid feature

2.777

$1.651-$

$<0.001$

4.669

$1.545 \quad 1.011-$

2.363

Perirenal fat infiltration

2.630

Pathology type

Clear cell RCC

$1.627-$
4.254

Reference $\quad 2.206$

-
$3.286-$
3.783

$<0.001$

0.045

2.698

1.541

4.809

0.001

Non-clear cell RCC

Nuclear grade

1.953

$1.236-$
3.085

Reference

0.098

$1-2$

0.004

3-4

Lymph node dissection

2.249

1.474

3.431

$<0.001$

1.981

$1.271-$

3.087

0.802

0.004

ASA grade

$\begin{array}{ll}0.657 & 0.302 \\ & 1.431\end{array}$

1
2
3

0.814

$0.320-$

Reference

HR: Hazard ratios; Cl: confidence interval; BMI: body mass index; IVC: inferior vena cava; RCC: renal cell carcinoma; ASA: American Society of Anesthesiologists; GADVR: growing against the direction of venous return 


\begin{tabular}{|c|c|c|c|c|c|c|}
\hline \multirow[b]{2}{*}{ Operative time, } & \multicolumn{3}{|c|}{ Univariate } & \multicolumn{3}{|c|}{ Multivariate } \\
\hline & 1.002 & $\begin{array}{l}1.001- \\
1.004\end{array}$ & 0.002 & - & - & 0.179 \\
\hline Surgical blood loss & 1.000 & $\begin{array}{l}1.000- \\
1.000\end{array}$ & 0.013 & - & - & 0.532 \\
\hline Postoperative complication & 1.509 & $\begin{array}{l}0.990- \\
2.298\end{array}$ & 0.055 & & & \\
\hline $\begin{array}{l}\text { Severe postoperative } \\
\text { complication }\end{array}$ & 1.833 & $\begin{array}{l}0.948- \\
3.543\end{array}$ & 0.071 & & & \\
\hline Thrombus adhering to the IVC & 1.297 & $\begin{array}{l}0.855- \\
1.968\end{array}$ & 0.221 & & & \\
\hline GADVR & 2.125 & $\begin{array}{l}1.124- \\
4.019\end{array}$ & 0.020 & 2.039 & $\begin{array}{l}1.030- \\
4.038\end{array}$ & 0.041 \\
\hline
\end{tabular}

The progression-free survival (PFS) was used as the prognostic outcome of this study. Univariate Cox regression analysis found that GADVR tumor thrombus, clinical symptoms, surgical approach, IVC segmental resection, postoperative serum creatinine, distant metastasis, tumor thrombus combined with bland thrombus, sarcomatoid feature, perirenal fat infiltration, pathological type, nuclear grade, lymph node dissection, operation time and surgical blood loss were the risk factors for PFS. Multivariate Cox regression analysis showed that GADVR tumor thrombus, symptoms, postoperative serum creatinine, distant metastasis, sarcomatoid feature, pathological type, lymph node dissection were independent risk factors for PFS. (See Table 2)

The survival curve analysis showed that the median PFS of patients with GADVR tumor thrombus was 14.0 months, while patients with non-GADVR tumor thrombus were 32.0 months $(P=0.016)$. GADVR tumor thrombus is an independent risk factor affecting the PFS for RCC and VTT (See Fig. 2). 
Table 3

Comparison of clinicopathological features between right RCC with GADVR and left RCC with GADVR.

\begin{tabular}{|c|c|c|c|}
\hline & $\begin{array}{l}\text { Right GADVR( } \mathrm{n}= \\
\text { 11) }\end{array}$ & $\begin{array}{l}\text { Left GADVR( } n= \\
\text { 10) }\end{array}$ & $\mathbf{p}$ \\
\hline Gender, n (\%) & $9(81.8 \%)$ & $7(70.0 \%)$ & 0.635 \\
\hline male & $2(18.2 \%)$ & $3(30.0 \%)$ & \\
\hline \multicolumn{4}{|l|}{ female } \\
\hline Age, $y$, mean $\pm S D$ & $55.09 \pm 10.19$ & $60.40 \pm 6.69$ & 0.179 \\
\hline Clinical symptoms, n (\%) & $0(0 \%)$ & $1(10.0 \%)$ & 1.000 \\
\hline No & $8(72.7 \%)$ & $8(80.0 \%)$ & \\
\hline Local symptoms & $1(9.1 \%)$ & $0(0 \%)$ & \\
\hline Systemic symptoms & $2(18.2 \%)$ & $1(10.0 \%)$ & \\
\hline \multicolumn{4}{|l|}{ Both } \\
\hline $\mathrm{BMI}, \mathrm{kg} / \mathrm{m}^{2}$, mean $\pm \mathrm{SD}$ & $25.18 \pm 2.03$ & $24.69 \pm 3.32$ & 0.687 \\
\hline Surgical approach, n (\%) & $2(18.2 \%)$ & $3(30.0 \%)$ & 0.635 \\
\hline Laparoscope & $9(81.8 \%)$ & $7(70.0 \%)$ & \\
\hline \multicolumn{4}{|l|}{ Open } \\
\hline Mayo classification, n (\%) & $0(0 \%)$ & $2(20.0 \%)$ & 0.430 \\
\hline 0 & $1(9.1 \%)$ & $3(20.0 \%)$ & \\
\hline 1 & $7(63.6 \%)$ & $12(50.0 \%)$ & \\
\hline 2 & $3(27.3 \%)$ & $4(10.0 \%)$ & \\
\hline \multicolumn{4}{|l|}{3} \\
\hline IVC segmental resection, $\mathrm{n}$ (\%) & $2(18.2 \%)$ & $6(60.0 \%)$ & 0.080 \\
\hline No & $9(81.8 \%)$ & $4(40.0 \%)$ & \\
\hline \multicolumn{4}{|l|}{ Yes } \\
\hline Preoperative serum creatinine, $\mu \mathrm{mol} / \mathrm{L}$, mean \pm SD & $109.82 \pm 20.89$ & $99.40 \pm 17.50$ & 0.233 \\
\hline $\begin{array}{l}\text { Serum creatinine one week after operation, } \mu \mathrm{mol} / \mathrm{L} \text {, } \\
\text { median (IQR) }\end{array}$ & $96.00(45.00)$ & $97.50(49.00)$ & 0.654 \\
\hline Tumor diameter, $\mathrm{cm}$, mean $\pm \mathrm{SD}$ & $8.70 \pm 2.77$ & $8.12 \pm 2.32$ & 0.611 \\
\hline
\end{tabular}

GADVR: growing against the direction of venous return; BMl: body mass index; SD: standard deviation; IQR: interquartile range; RCC: renal cell carcinoma; ASA: American Society of Anesthesiologists; IVC: inferior vena cava. 


\begin{tabular}{|c|c|c|c|}
\hline & $\begin{array}{l}\text { Right GADVR }(\mathrm{n}= \\
11)\end{array}$ & $\begin{array}{l}\text { Left GADVR( } \mathrm{n}= \\
\text { 10) }\end{array}$ & $\mathbf{p}$ \\
\hline Clinical N stage, n (\%) & $4(36.4 \%)$ & $1(10.0 \%)$ & 0.311 \\
\hline cNO & $7(63.6 \%)$ & $9(90.0 \%)$ & \\
\hline \multicolumn{4}{|l|}{$\mathrm{cN} 1$} \\
\hline Adrenal metastasis, $\mathrm{n}(\%)$ & $10(90.9 \%)$ & $8(80.0 \%)$ & 0.586 \\
\hline No & $1(9.1 \%)$ & $2(20.0 \%)$ & \\
\hline \multicolumn{4}{|l|}{ Yes } \\
\hline Distant metastasis, n (\%) & $11(100 \%)$ & $6(60.0 \%)$ & 0.035 \\
\hline No & $0(0 \%)$ & $4(40.0 \%)$ & \\
\hline \multicolumn{4}{|l|}{ Yes } \\
\hline Bland thrombus, n (\%) & $2(18.2 \%)$ & $7(70.0 \%)$ & 0.030 \\
\hline No & $9(81.8 \%)$ & $3(30.0 \%)$ & \\
\hline \multicolumn{4}{|l|}{ Yes } \\
\hline Sarcomatoid feature, n (\%) & $9(81.8 \%)$ & $9(90.0 \%)$ & 1.000 \\
\hline No & $2(18.2 \%)$ & $1(10.0 \%)$ & \\
\hline \multicolumn{4}{|l|}{ Yes } \\
\hline Perirenal fat infiltration, $\mathrm{n}(\%)$ & $8(72.7 \%)$ & $7(70.0 \%)$ & 1.000 \\
\hline No & $3(27.3 \%)$ & $3(30.0 \%)$ & \\
\hline \multicolumn{4}{|l|}{ Yes } \\
\hline Pathology type, n (\%) & $10(90.9 \%)$ & $7(70.0 \%)$ & 0.311 \\
\hline Clear cell RCC & $1(9.1 \%)$ & $3(30.0 \%)$ & \\
\hline \multicolumn{4}{|l|}{ Non-clear cell RCC } \\
\hline Nuclear grade, n (\%) & $4(36.4 \%)$ & $4(40.0 \%)$ & 1.000 \\
\hline 2 & $6(54.5 \%)$ & $5(50.0 \%)$ & \\
\hline 3 & $1(9.1 \%)$ & $1(10.0 \%)$ & \\
\hline 4 & & & \\
\hline
\end{tabular}

GADVR: growing against the direction of venous return; BMI: body mass index; SD: standard deviation; IQR: interquartile range; RCC: renal cell carcinoma; ASA: American Society of Anesthesiologists; IVC: inferior vena cava. 


\begin{tabular}{|c|c|c|c|}
\hline & $\begin{array}{l}\text { Right GADVR(n= } \\
11)\end{array}$ & $\begin{array}{l}\text { Left GADVR( } n= \\
10)\end{array}$ & p \\
\hline Lymph node dissection, n (\%) & $6(54.5 \%)$ & $6(60.0 \%)$ & 1.000 \\
\hline No & $5(45.5 \%)$ & $4(40.0 \%)$ & \\
\hline \multicolumn{4}{|l|}{ Yes } \\
\hline ASA grade, $\mathrm{n}(\%)$ & $1(9.1 \%)$ & $1(10.0 \%)$ & \multirow[t]{4}{*}{0.724} \\
\hline 1 & $8(72.7 \%)$ & $9(90.0 \%)$ & \\
\hline 2 & $2(18.2 \%)$ & $0(0 \%)$ & \\
\hline \multicolumn{3}{|l|}{3} & \\
\hline Operative time, min, mean \pm SD & $338.18 \pm 54.97$ & $404.30 \pm 125.70$ & 0.150 \\
\hline Surgical blood loss, mL, median (IQR) & $1500.00(1100.00)$ & $1000.00(650.00)$ & 0.085 \\
\hline Postoperative complication, n (\%) & $4(36.4 \%)$ & $6(60.0 \%)$ & 0.395 \\
\hline No & $7(63.6 \%)$ & $4(40.0 \%)$ & \\
\hline \multicolumn{4}{|l|}{ Yes } \\
\hline Severe postoperative complication, $n(\%)$ & $10(90.9 \%)$ & $9(90.0 \%)$ & \multirow[t]{3}{*}{1.000} \\
\hline No & $1(9.1 \%)$ & $1(10.0 \%)$ & \\
\hline \multicolumn{3}{|l|}{ Yes } & \\
\hline Thrombus adhering to the IVC, $\mathrm{n}(\%)$ & $1(9.1 \%)$ & $3(30.0 \%)$ & \multirow[t]{3}{*}{0.311} \\
\hline No & $10(90.9 \%)$ & $7(70.0 \%)$ & \\
\hline \multicolumn{3}{|l|}{ Yes } & \\
\hline $\begin{array}{l}\text { GADVR: growing against the direction of } \\
\text { deviation; IQR: interquartile range; RCC: re } \\
\text { Anesthesiologists; IVC: inferior vena cava }\end{array}$ & $\begin{array}{l}\text {; BMI: body mass } \\
\text { ioma; ASA: Ameri }\end{array}$ & $\begin{array}{l}\text { dex; SD: standard } \\
\text { i Society of }\end{array}$ & \\
\hline
\end{tabular}

Among patients with GADVR tumor thrombus, we divided them into right and left based on the primary renal tumor's side, with 11 cases and 10 cases, respectively. Bilateral renal anatomy is different. The right renal vein usually has no branch, so the tumor thrombus usually grows to the left renal vein against the venous return. Most patients' right gonadal vein flows directly into the inferior vena cava, and a few patients may mutate and flow into the right renal vein. Therefore, the GADVR tumor thrombus of right renal cancer may include the right gonadal vein tumor thrombus. The left renal vein usually has the left adrenal vein, the gonadal vein, the lumbar ascending vein as the branch, and the left GADVR tumor thrombus mainly manifests as the branch venous tumor thrombus. Comparison of clinicopathological features between right RCC with GADVR tumor thrombus and left RCC with GADVR tumor thrombus is shown in Table 3. We further classified GADVR tumor thrombus according to the different involved veins. Among the 11 patients with right GADVR tumor thrombus, ten patients $(90.9 \%)$ extended to the left renal 
vein tumor thrombus, and one patient (9.1\%) extended to the right gonadal vein. Among the ten patients with left GADVR tumor thrombus, there were one patient (10\%) with right renal vein, one patient (10\%) with left gonadal vein tumor thrombus, two patients $(20 \%)$ with left adrenal vein tumor thrombus, two patients $(20 \%)$ with lumbar ascending vein tumor thrombus, and four patients $(40 \%)$ with two or more branches of tumor thrombus. In the above ten patients with left GADVR tumor thrombus, there were 15 venous tumor thrombus growing against the direction of venous return, including four $(26.7 \%)$ in the left adrenal vein, five (33.3\%) in the left gonadal vein, four $(26.7 \%)$ in left lumbar ascending vein and two $(13.3 \%)$ in the right renal vein. (See Fig. 3)

\section{Discussion}

In the clinical practice, we found a particular type of patients whose tumor thrombus is growing against the direction of venous return. We define it as GADVR tumor thrombus(Tumor thrombus growing against the direction of venous return). Veins are blood vessels that carry blood back to the heart. The ventricles draw blood from the atria and large veins during diastole. When inhaling, the pleural cavity's negative pressure increases, and the pressure in the large veins in the thoracic cavity decreases, thereby promoting the return of venous blood(19). Through the role of heart and respiration, for the left kidney, blood flows from the branch of the left renal vein to the left renal vein and then enters the IVC. For the right kidney, blood flows from the right renal vein into the IVC. GADVR tumor thrombus is a characteristic feature of tumor thrombus, a secondary manifestation of rapid tumor growth or high malignancy. The malignant degree of tumors in these patients is often high, and it is easy to cause tumor thrombus to obstruct the IVC. The space in the inferior vena cava is limited, limiting the proliferation and growth of tumors. Therefore, the tumor thrombus extends to the branch vein or contralateral renal vein, showing an abnormal growth pattern against the direction of venous return. For the right RCC and VTT, the GADVR tumor thrombus is manifested as the left renal vein tumor thrombus. For left RCC and VTT, the GADVR tumor thrombus showed left adrenal vein, left gonadal vein (testicular or ovarian vein), and left lumbar ascending vein tumor thrombus. Although GADVR tumor thrombus is a secondary manifestation, the importance of this concept has not been emphasized or reflected in previous studies. We came up with this concept because we found that patients with this type of surgery are more complicated and have a worse prognosis in clinical practice.

In this study, we found that GADVR tumor thrombus incidence was $9.9 \%$ in all patients with RCC and VTT. In terms of surgical approach, $76.2 \%$ of patients with GADVR tumor thrombus chose open surgery, while the proportion of patients with non-GADVR tumor thrombus was $52.1 \%$. With the progress of minimally invasive technology, more and more centers have applied laparoscopic or robotic surgery, and the open approach is still a traditional and effective treatment. Our previous studies have found that the open 
approach is usually associated with large tumor load, severe adhesion, tumor thrombus invasion of the vascular wall, and full-filled tumor thrombus, which is a manifestation of complex surgery.(20)

This study found that GADVR tumor thrombus had a higher proportion of adhesion to the IVC and IVC segmental resection. In clinical practice, we found that GADVR tumor thrombus is easy to obstruct the IVC. After the tumor thrombus adheres to the IVC, the inferior vena cava space is limited, limiting tumor proliferation and growth. Therefore, the tumor thrombus extends to the branch vein or contralateral renal vein, showing an abnormal growth pattern against the direction of venous return. In previous studies, we found that tumor thrombus combined with bland thrombus was a risk factor for surgical complexity and poor prognosis(15), and bland thrombus was also a manifestation of obstructive tumor thrombus(21). We believe that the obstruction of tumor thrombus caused the slow blood flow, and the platelets and red blood cells in the blood gathered at the distal end of the tumor thrombus, resulting in long-term thrombosis; on the other hand, the obstruction caused the space limitation and formed the tumor thrombus against the direction of venous return. Therefore, it is considered that either tumor thrombus with bland thrombus or GADVR tumor thrombus formation is a secondary manifestation of tumor thrombus obstruction.

GADVR tumor thrombus has more operation time and more surgical blood loss in terms of surgical complexity, which requires clinicians to pay more attention. Before surgery, patients and their families should be fully communicated to inform them of surgical risks and get an understanding of patients. Although the incidence of severe complications was not significantly different from that of non-GADVR tumor thrombus, the overall incidence of postoperative complications was high, and more intensive care was needed after the operation.

In terms of prognosis, GADVR tumor thrombus is an independent risk factor affecting PFS. In this study, it was found that the median survival time of patients with GADVR tumor thrombus was 14.0 months, while that of patients with non-GADVR tumor thrombus was 32.0 months, and the difference was statistically significant. Patients with GADVR tumor thrombus should be followed up more closely after the operation.

We distinguished 21 patients with GADVR tumor thrombus according to the type of vein involved. Before surgery, we confirmed GADVR tumor thrombus's presence by urinary system enhanced CT or inferior vena cava enhanced MRI. Imaging findings usually show thickening of the branch vein with filling defect inside, and enhancement can be seen after the enhanced scan, which can be diagnosed as a branch tumor thrombus. Preoperative imaging is essential to determine the length of branch tumor thrombus to ensure sufficient resection of the involved vein. The operation will be as radical as possible to remove all tumor thrombus branches, along with the branch vein and its internal tumor thrombus, to ensure that the tumor resection clean, reduce the local recurrence rate. If necessary, a frozen pathological examination can be performed on the vein's stump to ensure that the vascular wall margin is negative.

This study has the following limitations: the amount of data included in this study is small, and a larger sample of long-term follow-up studies is needed for further verification. This paper is a single-center 
study and needs to be further verified by the subsequent inclusion of multi-center studies to ensure broad adaptability.

\section{Conclusions}

In summary, GADVR tumor thrombus is a characteristic feature of tumor thrombus, and its incidence is $9.9 \%$. The proportion of open surgical approach is relatively high and the operation is more complex, which is an independent risk factor affecting PFS.

\section{Declarations}

\section{Ethical Approval and consent to participate}

All procedures performed in studies involving human participants were in accordance with the ethical standards of the Peking University Third Hospital ethics committee. Informed consent was obtained from all individuals. This study was approved by the institutional review board of the Peking University Third Hospital (the number of the ethics approval: No.2018-396-01).

\section{Consent for publication}

Not applicable.

\section{Availability of data and materials}

The datasets used and/or analyzed during the current study are available from the corresponding author on reasonable request.

\section{Competing interests}

The authors declare that they have no competing interests.

\section{Funding}

This study was supported by the National Nature Science Foundation of China (NO. 81771842), National Nature Science Foundation of China (NO. 82072211).

\section{Authors' contributions}

ZL, YXL, LLM: study conception and design, literature search, clinical studies, data analysis, statistical analysis, manuscript preparation, manuscript editing. $X Z, L Y G, G D Z, P H, S Y T$ : study conception and design, literature search, clinical studies, data analysis, manuscript editing. SDZ, SMW, XJT, CL, HXZ: study conception and design, clinical studies, manuscript editing. LLM: guarantor of the integrity of the entire study. The authors have read and approved this manuscript, and ensure that the listed authors'contributions are accurate. 
Acknowledgments

The authors acknowledge Jing-Han Dong for her kind help with data collection and follow-up work, all the nurses in our center for their services, and patients involved in this study.

\section{Abbreviations}

GADVR: growing against the direction of venous return

RCC: renal cell carcinoma

VTT: venous tumor thrombus

PFS: progression-free survival

IVC: inferior vena cava

ASA: American Society of Anesthesiologists

HR: Hazard ratios

Cl: confidence interval

SD: standard deviation

IQR: interquartile range

BMl: body mass index

\section{References}

1. Chen W, Zheng R, Baade PD, Zhang S, Zeng H, Bray F, et al. Cancer statistics in China, 2015. CA Cancer J Clin. 2016;66:115-32.

2. Ljungberg B, Bensalah $K$, Canfield $S$, Dabestani $S$, Hofmann $F$, Hora $M$, et al. EAU guidelines on renal cell carcinoma: 2014 update. Eur Urol. 2015;67:913-24.

3. Abbasi A, Johnson TV, Ying K, Baumgarten D, Millner R, Master VA. Duplicated vena cava with tumor thrombus from renal cancer: use of venogram for safer operative planning. Urology. 2012;79:e57-e8.

4. Gu L, Li H, Wang Z, Wang B, Huang Q, Lyu X, et al. A systematic review and meta-analysis of clinicopathologic factors linked to oncologic outcomes for renal cell carcinoma with tumor thrombus treated by radical nephrectomy with thrombectomy. Cancer Treat Rev. 2018;69:112-20.

5. Manso M, Pacheco-Figueiredo L, Santos-Silva A, Silva J, Silva C, Cruz F. Renal Cell Carcinoma with Venous Thrombus: Should Surgery Be Offered When Metastasis Is Present at Diagnosis? Urol Int. 2018;101:387-90. 
6. Rodriguez Faba O, Linares E, Tilki D, Capitanio U, Evans CP, Montorsi F, et al. Impact of Microscopic Wall Invasion of the Renal Vein or Inferior Vena Cava on Cancer-specific Survival in Patients with Renal Cell Carcinoma and Tumor Thrombus: A Multi-institutional Analysis from the International Renal Cell Carcinoma-Venous Thrombus Consortium. European urology focus. 2018;4:435-41.

7. Nooromid MJ, Ju MH, Havelka GE, Kozlowski JM, Kundu SD, Eskandari MK. Fifteen-year experience with renal cell carcinoma with associated venous tumor thrombus. Surgery. 2016;160:915-23.

8. Agochukwu N, Shuch B. Clinical management of renal cell carcinoma with venous tumor thrombus. World J Urol. 2014;32:581-9.

9. Gu L, Ma X, Gao Y, Li H, Li X, Chen L, et al. Robotic versus Open Level I-II Inferior Vena Cava Thrombectomy: A Matched Group Comparative Analysis. J Urol. 2017;198:1241-6.

10. Bonsib SM, Bhalodia A. Retrograde venous invasion in renal cell carcinoma: a complication of sinus vein and main renal vein invasion. Mod Pathol. 2011;24:1578-85.

11. Gagné-Loranger M, Lacombe L, Pouliot F, Fradet V, Dagenais F. Renal cell carcinoma with thrombus extending to the hepatic veins or right atrium: operative strategies based on 41 consecutive patients. Eur J Cardiothorac Surg. 2016;50:317-21.

12. Nini A, Capitanio U, Larcher A, Dell'Oglio P, Dehò F, Suardi N, et al. Perioperative and Oncologic Outcomes of Nephrectomy and Caval Thrombectomy Using Extracorporeal Circulation and Deep Hypothermic Circulatory Arrest for Renal Cell Carcinoma Invading the Supradiaphragmatic Inferior Vena Cava and/or Right Atrium. Eur Urol. 2018;73:793-9.

13. Al-Katib S, Shetty M, Jafri SMA, Jafri SZH. Radiologic Assessment of Native Renal Vasculature: A Multimodality Review. Radiographics. 2017;37:136-56.

14. Blute ML, Leibovich BC, Lohse CM, Cheville JC, Zincke H. The Mayo Clinic experience with surgical management, complications and outcome for patients with renal cell carcinoma and venous tumour thrombus. BJU Int. 2004;94:33-41.

15. Liu Z, Zhang L, Hong P, Li L, Tang S, Zhao X, et al. The influence of venous tumor thrombus combined with bland thrombus on the surgical treatment and prognosis of renal cell carcinoma patients. Cancer Med. 2020;9:5860-8.

16. González J, Gorin MA, Garcia-Roig M, Ciancio G. Inferior vena cava resection and reconstruction: technical considerations in the surgical management of renal cell carcinoma with tumor thrombus. Urol Oncol. 2014;32:34.e19-34.e26.

17. Liu Z, Li L, Hong P, Zhu G, Tang S, Zhao X, et al. A Predictive Model for Tumor Invasion of the Inferior Vena Cava Wall Using Multimodal Imaging in Patients with Renal Cell Carcinoma and Inferior Vena Cava Tumor Thrombus. Biomed Res Int. 2020;2020:9530618.

18. Clavien PA, Barkun J, de Oliveira ML, Vauthey JN, Dindo D, Schulick RD, et al. The Clavien-Dindo classification of surgical complications: five-year experience. Ann Surg. 2009;250:187-96.

19. Repessé $X$, Charron C, Vieillard-Baron A. Assessment of the effects of inspiratory load on right ventricular function. Curr Opin Crit Care. 2016;22:254-9. 
20. Liu Z, Zhao X, Ge L, Wu B, Tang S, Hong P, et al. Completely laparoscopic versus open radical nephrectomy and infrahepatic tumor thrombectomy: Comparison of surgical complexity and prognosis. Asian J Surg. 2021;44:641-8.

21. Ayyathurai R, Garcia-Roig M, Gorin MA, González J, Manoharan M, Kava BR, et al. Bland thrombus association with tumour thrombus in renal cell carcinoma: analysis of surgical significance and role of inferior vena caval interruption. BJU Int. 2012;110:E449-E55.

\section{Figures}
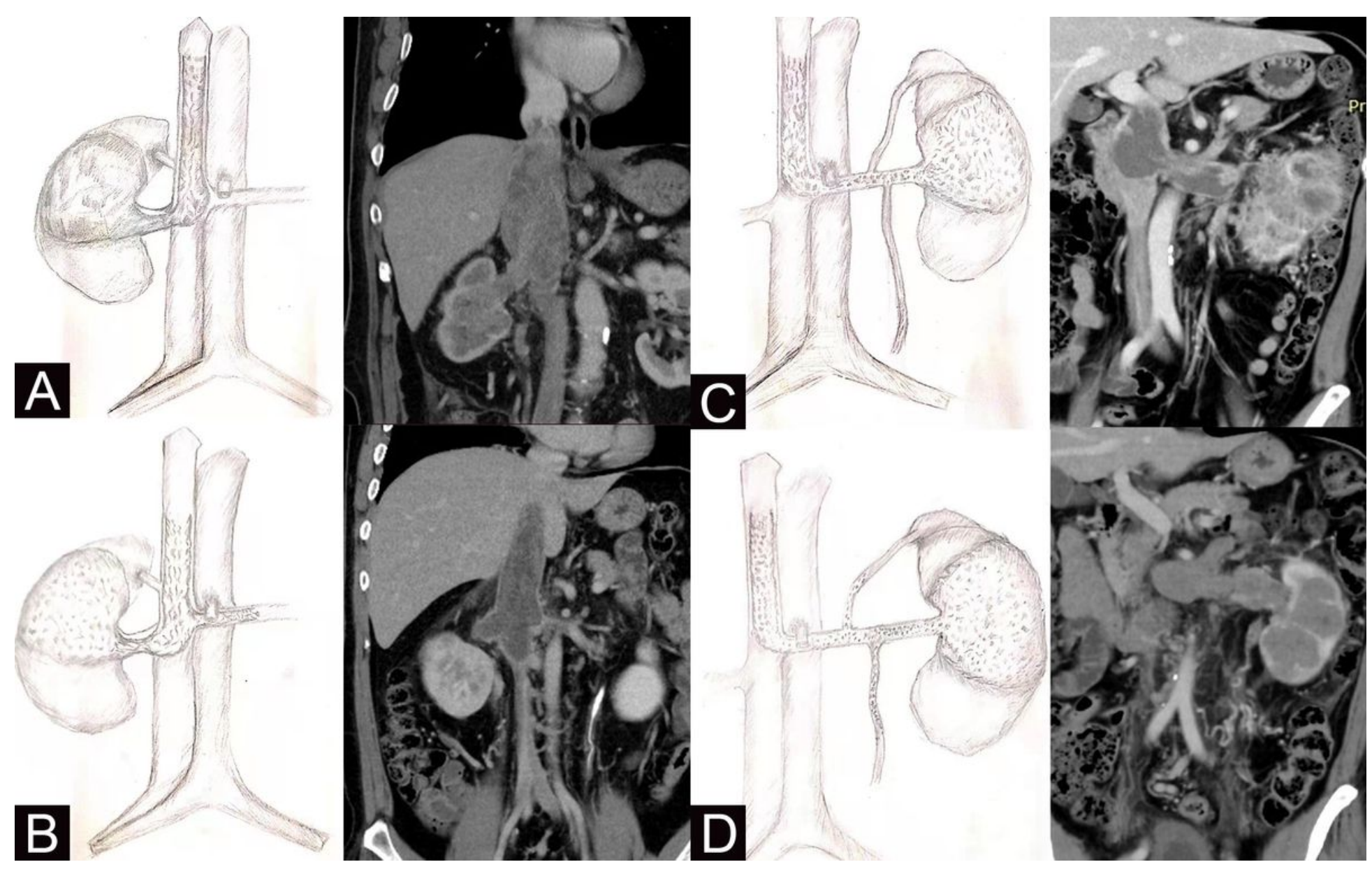

Figure 1

Morphological diagram of GADVR tumor thrombus and non-GADVR tumor thrombus and enhanced CT examination of the urinary system in typical patients (coronal position): The left image was diagram and the right image was enhanced CT examination of the urinary system (coronal position). A. Right RCC with IVC tumor thrombus without GADVR tumor thrombus: The tumor occurs from the right kidney, forming tumor thrombus in the right renal vein, and grows into the IVC with the direction of venous return. B. Right RCC with IVC tumor thrombus accompanied by GADVR tumor thrombus: The tumor occurs from the right kidney and forms tumor thrombus in the right renal vein. In addition to part of the tumor grows in the IVC with the direction of venous return, another part grows in the left renal vein against the direction of 
venous return or in the right gonadal vein against the direction of venous return. C. Left RCC with IVC tumor thrombus without GADVR tumor thrombus: The tumor occurs from the left kidney, forming tumor thrombus in the left renal vein, and grows into the IVC with the direction of venous return. D. Left RCC with IVC tumor thrombus accompanied by GADVR tumor thrombus: The tumor occurs from the left kidney and forms tumor thrombus in the left renal vein. In addition to part of the tumor grows into the IVC with the direction of venous return, another part of the tumor grows into the left renal vein branches such as the left adrenal vein, gonadal vein, and ascending lumbar vein, against the direction of venous return.

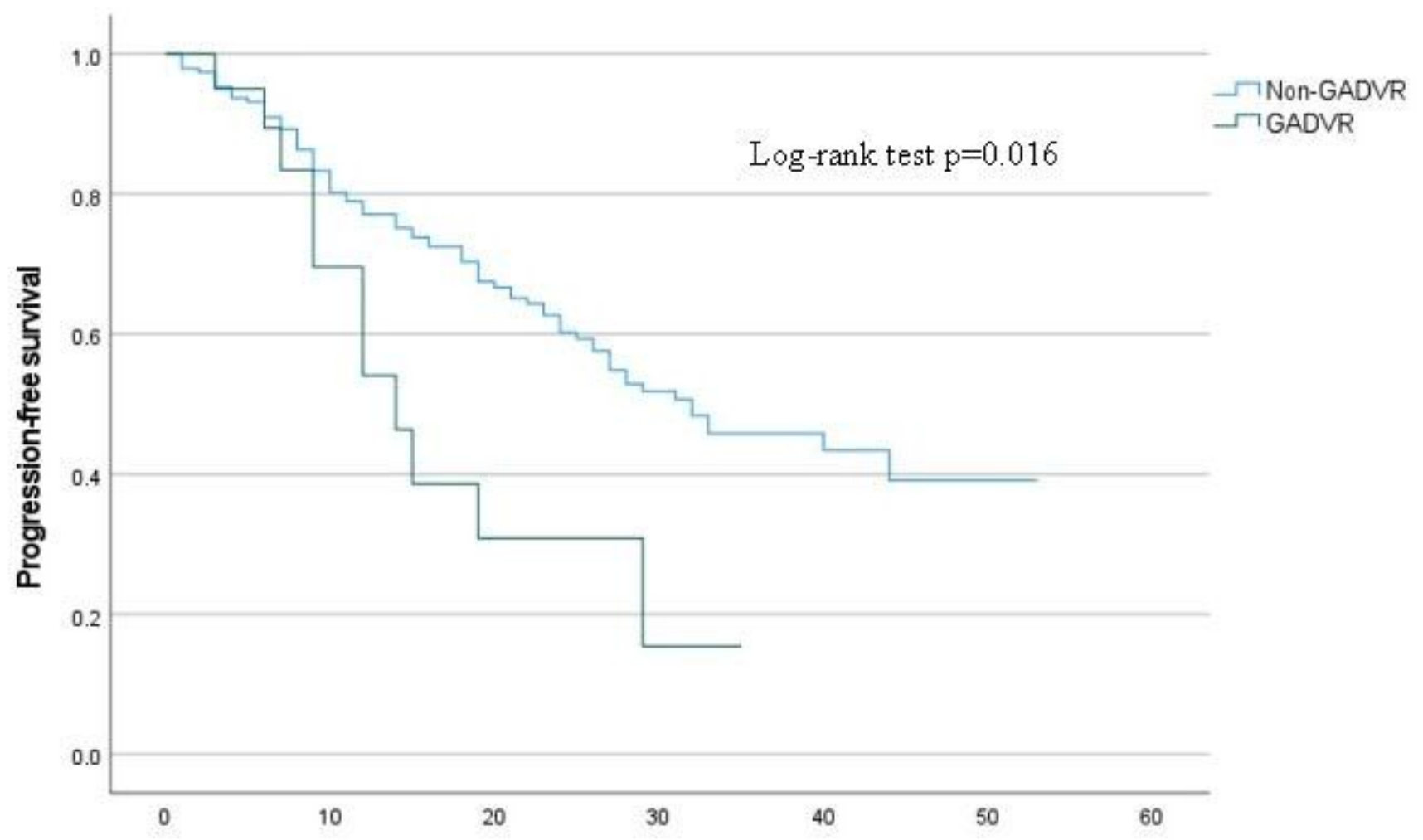

Following-up time (months)

Figure 2

Progression-free survival of GADVR tumor thrombus and non-GADVR tumor thrombus GADVR: growing against the direction of venous return

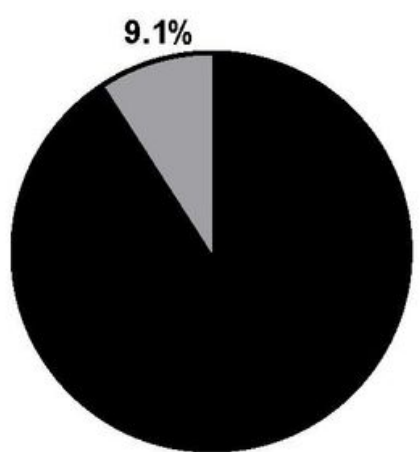

$90.9 \%$

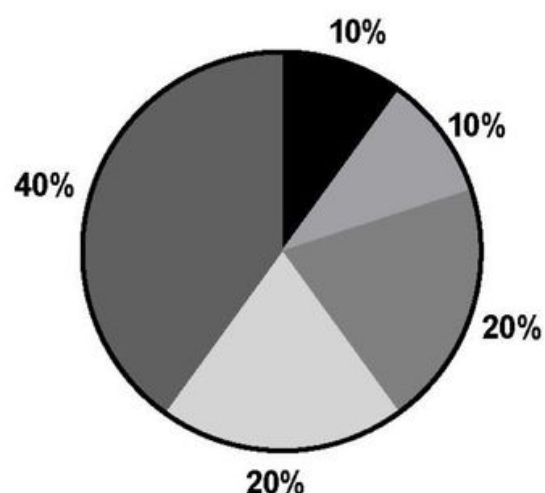

$20 \%$
Contralateral renal vein

Gonadal vein

Adrenal vein

Lumbar ascending vein

Two or more branches 
Figure 3

Types of affected veins in patients with GADVR tumor thrombus (pie chart) A. Patients with right GADVR tumor thrombus; B. Patients with left GADVR tumor thrombus. GADVR: growing against the direction of venous return 